\title{
Efficacy and safety of roxadustat in the treatment of renal allograft anemia patients: a case series
}

\author{
Jun $\mathrm{Li}^{1 /}$, Kuifen Ma ${ }^{1,2 \#}$, Liangping Wang ${ }^{3}$, Huaji $\mathrm{Qi}^{3},{\mathrm{Junhao} \mathrm{Lv}^{4} \text {, Yuefeng Rao }{ }^{1} \text {, Wenhan Peng }}^{4}$ \\ ${ }^{1}$ Department of Clinical Pharmacy, the First Affiliated Hospital, Zhejiang University School of Medicine, Hangzhou, China; ${ }^{2}$ Zhejiang Provincial \\ Key Laboratory for Drug Evaluation and Clinical Research, Hangzhou, China; ${ }^{3}$ Department of Pharmacy, Linping District Hospital of Integrated \\ Chinese and Western Medicine, Hangzhou, China; ${ }^{4}$ Kidney Disease Center, the First Affiliated Hospital, Zhejiang University School of Medicine, \\ Hangzhou, China \\ Contributions: (I) Conception and design: J Li, K Ma; (II) Administrative support: Y Rao, W Peng; (III) Provision of study materials or patients: W \\ Peng; (IV) Collection and assembly of data: L Wang, H Qi; (V) Data analysis and interpretation: K Ma, H Qi; (VI) Manuscript writing: All authors; \\ (VII) Final approval of manuscript: All authors. \\ \#These authors contributed equally to this work \\ Correspondence to: Wenhan Peng. Kidney Disease Center, the First Affiliated Hospital, Zhejiang University School of Medicine, Hangzhou 310003, \\ China. Email: 1198027@zju.edu.cn; Yuefeng Rao. Department of Clinical Pharmacy, the First Affiliated Hospital, Zhejiang University School of \\ Medicine, Hangzhou 310003, China. Email: raoyf@zju.edu.cn.
}

Background To observe the efficacy and safety of roxadustat, an inhibitor of proline hydroxylase, in renal allograft anemia patients.

Methods: This prospective study collected the clinical data of renal transplant patients treated with roxadustat for anemia at the Kidney Disease Center of the First Affiliated Hospital of Zhejiang University from April to August 2020. The patients were followed up every 2 weeks, and the changes in their hemoglobin index and any adverse reactions were recorded during 10 weeks of treatment. The efficacy of roxadustat for treatment of anemia after kidney transplantation was analyzed by comparing the change and increase in average hemoglobin levels before and after treatment. Rates of treatment response and achievement of the standard hemoglobin level were statistically analyzed. In addition, any potential adverse events and the glomerular filtration rate were recorded for 10 weeks to assess the safety of roxadustat in renal allograft anemia patients.

Results: After 10 weeks of roxadustat treatment, the mean hemoglobin level was $10.4 \pm 3.9 \mathrm{~g} / \mathrm{dL}$, which was significantly higher than at baseline. Over the entire period, treatment was observed to have a therapeutic effect at weeks 2-4, with mean hemoglobin levels increasing as treatment time increased. At the 10-week endpoint, the percentage of patients reaching the standard hemoglobin level and exhibiting a response to treatment was $52.4 \%$ and $71.4 \%$, respectively. During the treatment, there was no rejection, and the glomerular filtration rate was stable. Only one person showed symptoms of fatigue, and there were no other obvious adverse reactions reported.

Conclusions: Roxadustat significantly improves hemoglobin levels and can be safely used in renal transplant anemia patients.

Keywords: Roxadustat; renal transplantation; anemia; safety; efficacy

Submitted Sep 23, 2021. Accepted for publication Nov 16, 2021.

doi: 10.21037/apm-21-2916

View this article at: https://dx.doi.org/10.21037/apm-21-2916 


\section{Introduction}

Chronic kidney disease (CKD) is a global public health challenge that affects approximately $10 \%$ of the world's population, including 120 million people in China (1). Anemia is a common complication of CKD mainly due to kidney damage, which affects the secretion of erythropoietin (EPO) (2). The more severe the anemia, the higher the risk of mortality, cardiovascular events, and hospitalization (3). Kidney transplantation is one of the most effective methods for the treatment of end-stage renal disease. According to statistics from the Chinese Kidney Transplantation Scientific Register System, 112 nationwide transplant centers conduct kidney transplantations, and more than 10,000 kidney transplantation surgeries are performed every year (4). Anemia following kidney transplantation usually resolves within 3 months of the transplant, and the secretion of EPO gradually increases. In the majority of patients, anemia significantly improves following transplantation. However, anemia is slow to resolve for some kidney transplant patients for several reasons, including graft damage, systemic disease after transplantation, and chronic infection (5). Studies have shown that the incidence of anemia at 6 months and 5 years after kidney transplantation is $70 \%$ and $33.1 \%$, respectively $(6,7)$. Moreover, the serum creatinine clearance rate after renal transplantation has been positively correlated with hemoglobin, and the incidence of anemia was reported as $60.1 \%$ and $29 \%$ in patients with serum creatinine $>177$ and $<177 \mathrm{~mol} / \mathrm{L}$, respectively (8).

Erythropoiesis is stimulated by an accumulation of endogenous plasma EPO and suppression of hepcidin, an indirect regulator of iron absorption and utilization. When hepcidin levels are suppressed, intestinal absorption of iron increases, and red blood cell maturation is promoted (9). At present, the most common treatment for anemia is the use of recombinant human EPO (rHuEPO) and iron supplementation (10,11). Nevertheless, approximately $5-10 \%$ of patients with chronic nephrotic anemia demonstrate EPO resistance, requiring higher doses of EPO to achieve adequate hemoglobin levels, but which carries an increased risk of cardiovascular events, mortality, and other adverse events $(11,12)$. Roxadustat is an oral inhibitor of hypoxia-inducible factor (HIF) prolyl hydroxylase that stimulates erythropoiesis and regulates iron metabolism. Clinical trials have shown that roxadustat can be used to treat anemia in dialysis or nondialysis patients and is noninferior to EPO $(13,14)$. However, roxadustat's efficacy in patients with renal allograft anemia remains unclear. It is also unknown whether roxadustat affects renal function after transplantation or increases the risk of rejection after transplantation. Here, we report the clinical outcome of treatment with roxadustat in 21 patients with EPO resistance or a continuous drop in hemoglobin after renal transplantation, providing a new perspective for the treatment of renal allograft anemia.

We present the following article in accordance with the STROBE reporting checklist (available at https://dx.doi. org/10.21037/apm-21-2916).

\section{Methods}

\section{Patients}

From April 2020 to August 2020, we collected the clinical data of patients with renal allograft anemia admitted to the First Affiliated Hospital of Zhejiang University who commenced treatment with roxadustat for 10 weeks.

The inclusion criteria were as follows: (I) patients admitted to hospital for complications after renal transplantation; (II) patients with anemia, defined as a hemoglobin level $<10 \mathrm{~g} / \mathrm{dL}$ (13); (III) patients who commenced treatment with roxadustat after admission; (IV) patients who were agreeable to be followed up by medical staff. Patients were excluded for the following reasons: (I) individuals whose hemoglobin levels were $\geq 10 \mathrm{~g} / \mathrm{dL}$ when first treated with roxadustat; (II) cases who were participating in, or had participated in, other clinical trials within the previous 3 months.

\section{Data collection}

The patients' general medical records were collected, including sex, age, body weight, time since transplantation, baseline hemoglobin value, epidermal growth factor receptor (eGFR), transferrin saturation, ferritin, C-reactive protein, and presence of diabetes mellitus or hypertension. Determination of EPO resistance was made according to the criteria recommended by the Chinese expert consensus on diagnosis and treatment of renal anemia (15), which specify a nil increase in hemoglobin levels compared with baseline values even after a month of treatment with EPO at $300 \mathrm{IU} / \mathrm{kg} /$ week. Roxadustat was given orally three times a week (TIW), with a weight-based starting dose of $70 \mathrm{mg}$ (in patients weighing 40 to $<60 \mathrm{~kg}$ ), $100 \mathrm{mg}$ (in patients weighing $\geq 60 \mathrm{~kg}$ ), or $120 \mathrm{mg}$ (in patients weighing $\geq 80 \mathrm{~kg}$ ) 
for nondialysis patients. The attending doctor adjusted the dose according to changes observed in hemoglobin levels during treatment. From week 2 to 10, mean hemoglobin levels were assessed once every 2 weeks and compared with baseline mean levels. The endpoint of treatment was 10 weeks. Based on the European Renal Best Practice (ERBP) guidelines (16), if the fortnightly hemoglobin index showed an increase $\geq 1 \mathrm{~g} / \mathrm{dL}$ compared with the baseline value, it was considered a treatment response. Conversely, an increase $<1 \mathrm{~g} / \mathrm{dL}$ was considered as no response. As a consequence of treatment, a patient achieving a hemoglobin level of $10-12 \mathrm{~g} / \mathrm{dL}$ was considered to have reached the standard level. The number of patients who reached the standard level and who had a response to treatment was recorded.

\section{Statistical analysis}

Descriptive statistics are reported as the mean \pm SD for continuous variables and the number of cases (n, \%) for categorical variables. All cases were included. Patients who discontinued roxadustat for EPO treatment were excluded from the hemoglobin improvement analysis. The Student's $t$-test was used to compare continuous variables between groups, and a $\mathrm{P}$ value $<0.05$ was considered statistically significant.

\section{Ethical statement}

All procedures performed in this study involving human participants were in accordance with the Declaration of Helsinki (as revised in 2013). The study was approved by Clinical research ethics committee of the First Affiliated Hospital, Zhejiang University School of Medicine (No. 2020-652) and informed consent was taken from all the patients.

\section{Results}

\section{Patient baseline characteristics}

From April to August 2020, a total of 21 patients with renal allograft anemia received oral roxadustat treatment, including 10 men and 11 women aged 16-62 years. The average time since kidney transplantation was $6.0 \pm 8.4$ years. The baseline mean hemoglobin level was $6.9 \pm 2.2 \mathrm{~g} / \mathrm{dL}$, and $28.6 \%$ of patients had a hemoglobin level $\geq 8.0 \mathrm{~g} / \mathrm{dL}$. The mean glomerular filtration rate was $28.8 \pm 16.1 \mathrm{~mL} / \mathrm{min} / 1.73 \mathrm{~m}^{2}$. Transferrin saturation $\geq 20 \%$ was recorded for $90 \%$ of patients; $60 \%$ exhibited ferritin levels $\geq 200 \mathrm{~g} / \mathrm{L}$, and $28.6 \%$ recorded C-reactive protein levels higher than the upper limit of the normal range. In addition, $23.8 \%$ of patients had diabetes, and $80.9 \%$ had hypertension (Table 1).

\section{Analysis of the efficacy of roxadustat}

\section{Clinical characteristics of included cases and evaluation of clinical effects}

Of the 21 patients with renal allograft anemia, 11 patients were EPO resistant, 9 patients were simultaneously treated with polysaccharide iron capsules, 6 patients were treated with EPO (6,000 IU, TIW), and 6 patients had C-reactive protein levels $>8 \mathrm{mg} / \mathrm{L}$. The baseline mean level of ferritin was $333.5 \pm 322.4 \mu \mathrm{g} / \mathrm{L}$, and the average ferritin level was $539.2 \pm 741.1 \mu \mathrm{g} / \mathrm{L}$ after 10 weeks $(\mathrm{P}>0.05)$.

The transplant complications included renal insufficiency, graft failure, delayed graft function (DGF), and infection at various sites. At the end of 10 weeks, 15 patients had responded to treatment, and 11 patients had reached the standard hemoglobin level. Six patients had no response, and their complications were as follows: chronic rejection leading to renal graft failure (Case 3), renal graft insufficiency (Cases 6 and 7), DGF (Case 15), microvirus (Case 18), and urinary tract infection with kidney stones (Case 20). It should be noted that 11 patients stopped the drug within 10 weeks. Of these, 3 patients discontinued roxadustat due to nonresponse after 6 weeks of treatment, 5 cases reached the standard hemoglobin level and then stopped, and another 3 cases had response at first but stopped, in which, 2 patients (Cases 13 and 14) thought roxadustat was increasing hemoglobin too slowly and requested to switch to erythrophin (Table 2).

\section{Hemoglobin improvement}

On initiation of treatment with roxadustat, the mean hemoglobin level was $6.9 \pm 2.2 \mathrm{~g} / \mathrm{dL}$ and increased to $10.4 \pm 3.9 \mathrm{~g} / \mathrm{dL}$ at 10 weeks of treatment $(\mathrm{P}<0.05)$. Compared with baseline, the hemoglobin levels at each fortnightly assessment after onset of treatment were significantly improved (Figure 1). The EPO-resistant group had a mean hemoglobin level of $6.9 \pm 2.8 \mathrm{~g} / \mathrm{dL}$ at baseline and $10.5 \pm 4.7 \mathrm{~g} / \mathrm{dL}$ at 10 weeks of treatment. In the EPOnonresistant group, the mean hemoglobin level at baseline was $6.8 \pm 1.1$ and $10.3 \pm 2.4 \mathrm{~g} / \mathrm{dL}$ at 10 weeks of treatment. There was no significant difference between the two groups (Table 3). 
Table 1 Baseline demographic, clinical, and laboratory characteristics of included patients $(n=21)$

\begin{tabular}{|c|c|}
\hline Characteristics & $\begin{array}{l}\text { Treated with roxadustat } \\
\qquad(\mathrm{n}=21)\end{array}$ \\
\hline Age (years), mean \pm SD & $45.2 \pm 11.8$ \\
\hline Male sex (\%) & 47.6 \\
\hline Weight $(\mathrm{kg})$, mean \pm SD & $58.3 \pm 9.3$ \\
\hline \multicolumn{2}{|c|}{ Time since transplant (months) } \\
\hline Mean \pm SD & $6.0 \pm 8.4$ \\
\hline$\geq 6(\%)$ & 47.6 \\
\hline$<6(\%)$ & 52.4 \\
\hline \multicolumn{2}{|l|}{ Hemoglobin (g/dL) } \\
\hline Mean \pm SD & $6.9 \pm 2.2$ \\
\hline$\geq 8.0(\%)$ & 28.6 \\
\hline$<8.0(\%)$ & 71.4 \\
\hline \multicolumn{2}{|l|}{ eGFR (mL/min/1.73 m²) } \\
\hline Mean \pm SD & $28.8 \pm 16.1$ \\
\hline$<10(\%)$ & 19.0 \\
\hline$\geq 10$ and $<30(\%)$ & 48.0 \\
\hline$\geq 30(\%)$ & 33.0 \\
\hline \multicolumn{2}{|l|}{ Transferrin saturation } \\
\hline Mean \pm SD (\%) & $51.1 \pm 27.9$ \\
\hline$<20 \%(\%)$ & 10.0 \\
\hline$\geq 20 \%(\%)$ & 90.0 \\
\hline \multicolumn{2}{|l|}{ Ferritin $(\mu \mathrm{g} / \mathrm{L})$} \\
\hline Mean \pm SD & $333.5 \pm 322.4$ \\
\hline$<100(\%)$ & 25.0 \\
\hline$\geq 100$ and $<200(\%)$ & 15.0 \\
\hline$\geq 200$ (\%) & 60.0 \\
\hline \multicolumn{2}{|l|}{ C-reactive protein (mg/L) } \\
\hline$\leq 8(\%)$ & 71.4 \\
\hline$>8(\%)$ & 28.6 \\
\hline Diabetes (\%) & 23.8 \\
\hline Hypertension (\%) & 80.9 \\
\hline
\end{tabular}

eGFR, epidermal growth factor receptor.

\section{Standard hemoglobin level attainment and treatment response rates}

The number of patients who reached the standard hemoglobin level at 2, 4, 6, 8, and 10 weeks were 6, 6, 9, 9, and 11 , respectively; the number of patients who showed a response at 2, 4, 6, 8, and 10 weeks of treatment were 10 , $15,15,16$, and 15, respectively. Two patients changed from response to no response during the treatment due to an exacerbation of their microvirus infection, which affected the hemoglobin level. The therapeutic effect of roxadustat treatment occurred in weeks $2-4$, and by week 10, 52.4\% of all patients had attained the standard hemoglobin level and $71.4 \%$ had shown a treatment response (Figure 2). In the EPO-resistant subgroup, $63.6 \%$ of patients had reached the standard hemoglobin level and $72.7 \%$ had achieved treatment response at 10 weeks, and $40 \%$ and $70 \%$ of the EPO-nonresistant group had reached the standard hemoglobin level and achieved a treatment response, respectively. These results demonstrate that roxadustat has a good therapeutic effect in both EPO-resistant and nonresistant patients (Table 4).

\section{Safety analysis}

At each follow-up, we collected adverse event information using open questions. The focus was on patients who reported any diarrhea, fever, respiratory tract infection, hyperkalemia, metabolic acidosis, fatigue, headache, rash, or other adverse events. Only one patient reported fatigue symptoms during the treatment period. This patient had a good improvement in hemoglobin and finally reached the treatment target. The remainder of the patients reported no significant adverse events. All patients had regular liver function reviews, and no abnormal liver enzyme indexes were recorded. In addition, roxadustat did not cause rejection after renal transplantation. During the treatment period, the glomerular filtration rate of the 21 patients did not deteriorate and even increased slightly (Figure 3).

\section{Discussion}

In this study of 21 patients, treatment with roxadustat began to take effect after 2-4 weeks. As the duration of treatment 


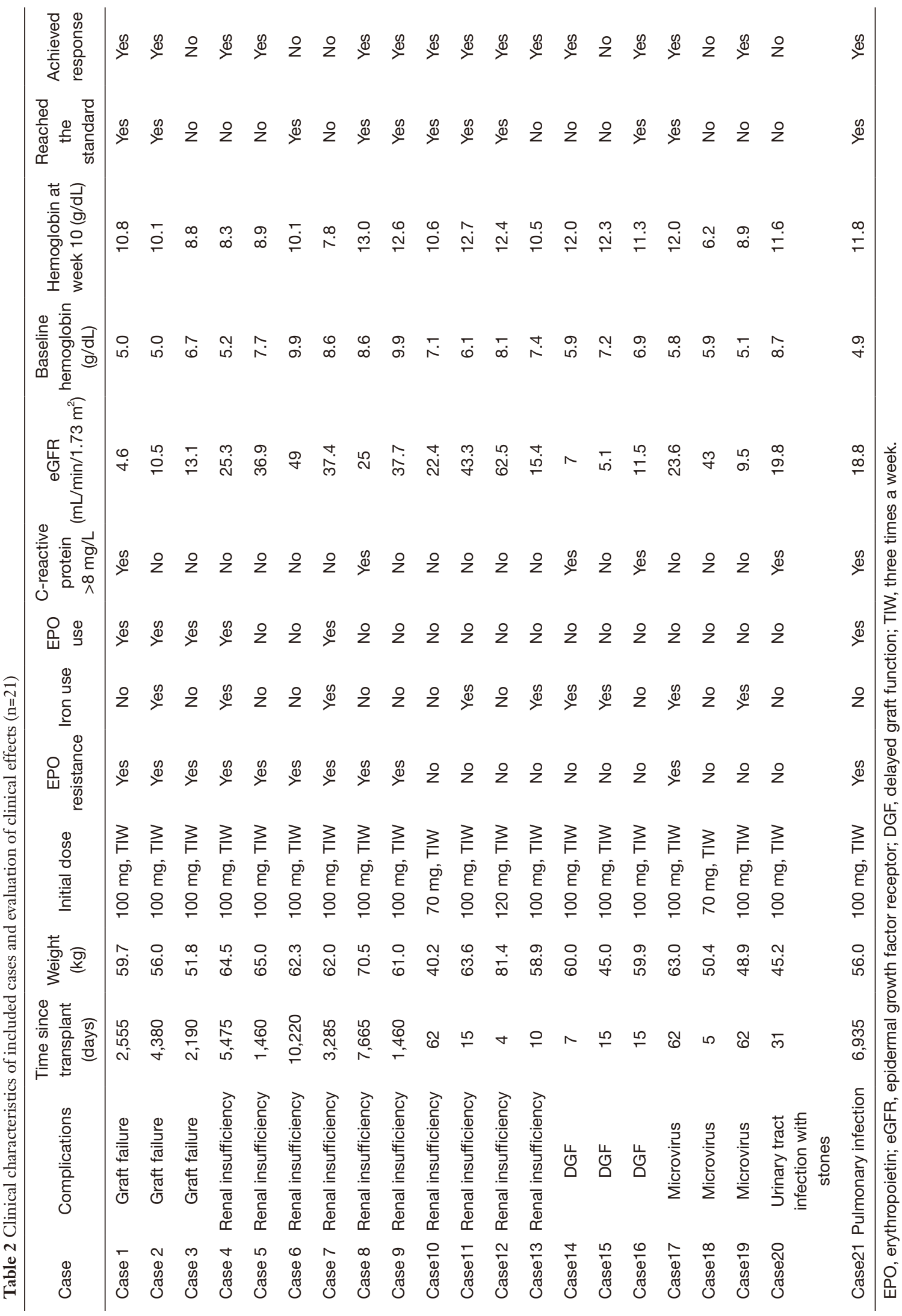




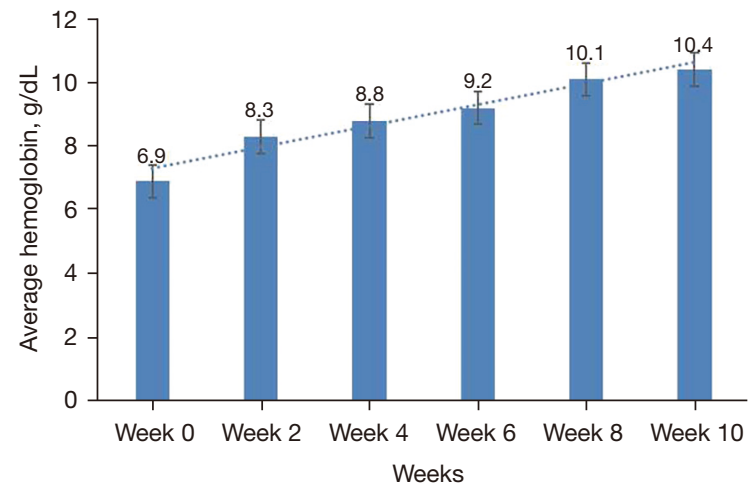

Figure 1 Changes in mean hemoglobin levels $(n=21)$.

Table 3 Hemoglobin status of EPO-resistant and nonresistant patients $(\mathrm{n}=21)$

\begin{tabular}{lcc}
\hline Group & $\begin{array}{c}\text { Baseline } \\
\text { hemoglobin }(\mathrm{g} / \mathrm{dL})\end{array}$ & $\begin{array}{c}\text { Hemoglobin at } \\
\text { week 10 }(\mathrm{g} / \mathrm{dL})\end{array}$ \\
\hline EPO-resistant patients & $6.9 \pm 2.8$ & $10.5 \pm 4.7$ \\
EPO-nonresistant patients & $6.8 \pm 1.1$ & $10.3 \pm 2.4$ \\
\hline
\end{tabular}

Data were reported as mean \pm SD. EPO, erythropoietin.

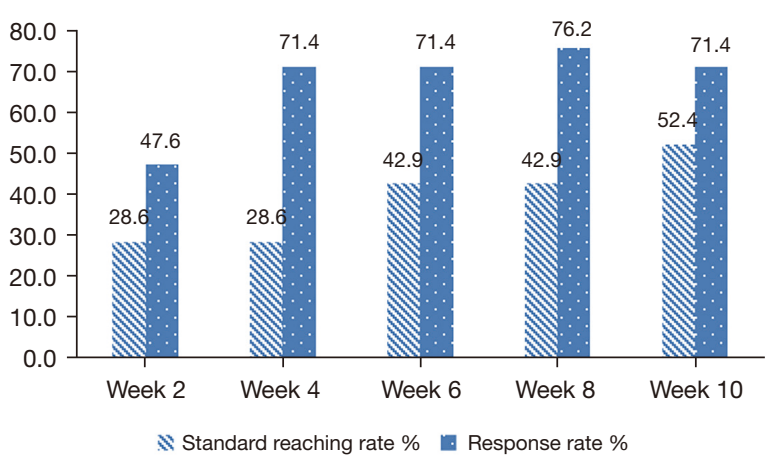

Figure 2 Changes in the standard hemoglobin level and treatment response rates after roxadustat treatment $(n=21)$.

Table 4 Attainment of the standard hemoglobin level and treatment response rates in EPO-resistant and EPO-nonresistant patients $(n=21)$

\begin{tabular}{lcc}
\hline Group & $\begin{array}{c}\text { Standard hemoglobin } \\
\text { level at week 10 }(\%)\end{array}$ & $\begin{array}{c}\text { Response rate } \\
\text { at week 10 (\%) }\end{array}$ \\
\hline EPO-resistant patients & 63.6 & 72.7 \\
EPO-nonresistant patients & 40.0 & 70.0 \\
\hline
\end{tabular}

EPO, erythropoietin. increased, so did the mean hemoglobin level. At the 10 -week endpoint, $52.4 \%$ of patients had reached the standard hemoglobin level, and the treatment response rate was $71.4 \%$. The endpoint of this study was 10 weeks, and the number of people who reached the standard hemoglobin level and showed a treatment response was recorded every 2 weeks. However, the duration of time that should be used to judge whether roxadustat treatment is effective or whether there are better predictors of treatment effectiveness remain issues worthy of further study.

Previous studies have reported that anemia following kidney transplantation can be divided into early and late stages at the critical 6-month period following renal transplantation. Early anemia is most commonly linked to intraoperative blood loss, frequent blood sampling tests, rejection, DGF, and drug-induced bone marrow suppression, while late anemia is mainly attributed to reduced allograft renal function, infections, chronic inflammatory states, and immunosuppressant and antiviral drugs $(17,18)$. The present study consisted of 21 patients with renal allograft anemia that was difficult and complicated to treat. Eleven patients had EPO resistance before using roxadustat, and 3 of those patients still had no response after combination or conversion to roxadustat.

We also analyzed the effectiveness of roxadustat in the EPO-resistant and nonresistant subgroups. The percentage of EPO-resistant patients who reached the standard hemoglobin level and exhibited a response to treatment at 10 weeks was $63.6 \%$ and $72.7 \%$, respectively; in the nonresistant EPO group, the percentages were $40 \%$ and $70 \%$, respectively, with no significant difference between the two groups. These results indicate that EPO resistance is not an important factor in the efficacy of roxadustat.

Infection, microviruses, BK viruses, and cytomegalovirusherpes zoster virus caused by the immunosuppressive state after renal transplantation are also associated with an increased risk of anemia $(5,19)$. In addition, the increase of plasma C-reactive protein concentration is related to the decrease in hemoglobin. Inflammation mainly stimulates the synthesis of fermodulin in the liver through the inflammatory cytokine IL-6, leading to iron deficiency and the inability of red blood cells to mature normally $(20,21)$. During EPO treatment, a previous study found that patients with high C-reactive protein had a lower hemoglobin response (22). However, roxadustat can correct the iron metabolism disorder caused by inflammation in 


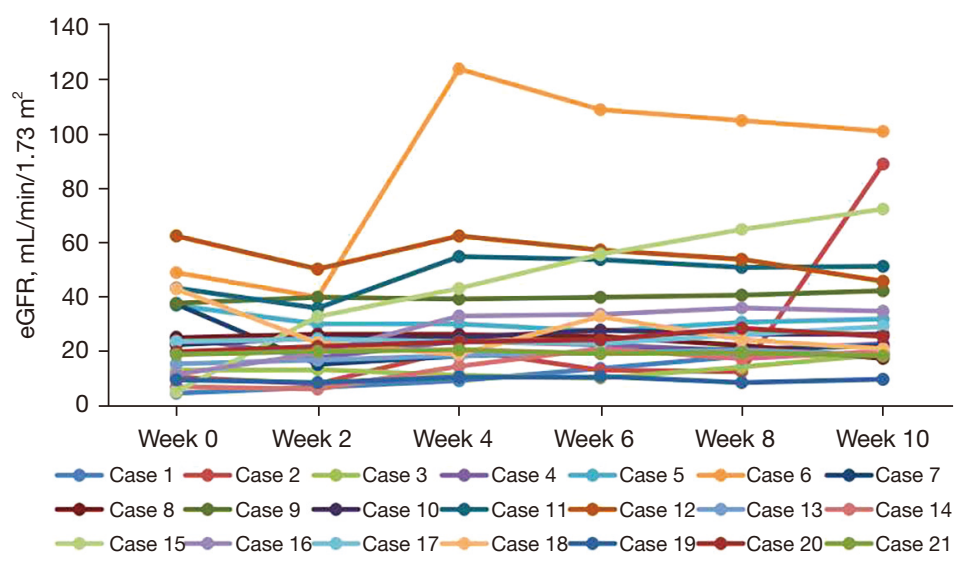

Figure 3 Glomerular filtration rate during roxadustat treatment $(n=21)$. eGFR, epidermal growth factor receptor.

the body and is not affected by C-reactive protein, making it possible to coordinate the formation of red blood cells by reducing iron modulin $(23,24)$. Among the 6 patients with high C-reactive protein, only 1 had no response to treatment. This patient had a urinary tract infection that was possibly associated with oxalate crystal deposition and acute renal damage following kidney transplantation (Case 20), Thus, roxadustat is effective in treating inflammatory infections after renal transplantation, but the efficacy is not ideal for patients with inflammatory conditions who also have impaired renal function. The results of roxadustat treatment for anemia patients with microvirus infections after kidney transplantation showed that one patient reached the standard hemoglobin level (Case 17). This patient's microvirus DNA was reduced from $1.1 \times 10^{9}$ to $4.1 \times 10^{4}$ copies $/ \mathrm{mL}$, and continued to improve after discharge. Two patients showed no significant improvement in microvirus indicators (Cases 18 and 19). Their treatment effects were not stable during the observation period, with some individual observation points decreasing from response to no response. It has been reported that immunoglobulin (IVIG) therapy combined with EPO to treat microvirus infections (HPV-B19) after renal transplantation could result in pure erythrocytic proliferative anemia (25). However, the results are controversial. Under normal conditions, EPO in plasma promotes the differentiation of bone marrow hematopoietic stem cells into erythroid progenitor cells and the gradual formation of mature new erythrocytes (26). In addition to inhibiting the bone marrow, microviruses also display characteristics of eosinophils, which replicate in large quantities after entering erythroid progenitor cells and induce apoptosis (27). Therefore, we believe that the most important therapeutic target for anemia caused by microvirus infections is the eradication of viremia. Continuous IVIG treatment $(0.4-1.0 \mathrm{~g} / \mathrm{kg} / \mathrm{d})$ for 5 days combined with roxadustat symptomatic treatment may be a better choice than EPO for improving anemia. For patients with renal allograft insufficiency or DGF within 2 weeks of transplantation (Cases 11-16), the rapid improvement in anemia was caused by the administration of roxadustat and the gradual recovery of renal function. Only 1 patient with delayed renal allograft rehabilitation (Case 15; creatinine $904 \mu \mathrm{mol} / \mathrm{L})$ had no response. Another 3 unresponsive patients were Case 6 (28 years after renal transplantation with renal allograft insufficiency), Case 7 ( 9 years after renal transplantation with renal allograft insufficiency), and Case 3 (6 years after renal transplantation with graft failure due to irregular immunosuppressant use, resulting in rejection; creatinine $448 \mu \mathrm{mol} / \mathrm{L}$ ). The HIF is a type of DNA-binding transcription factor. When oxygen levels drop, it can activate the expression of the EPO gene and participates in erythropoiesis, iron regulation, angiogenesis, etc. Under normal oxygen conditions, HIF will degrade rapidly and silence the hypoxia response gene $(28,29)$. The causes of delayed renal function and renal insufficiency are multiple, including both immune and non-immune mechanisms of renal injury. The failure of HIF-prolyl hydroxylase inhibitor (PHI) may be due to the "switching phenomenon" of EPO-producing cells. It may also be related to the degree of allograft insufficiency and a long history of renal transplantation.

Roxadustat is an oral HIF-PHI stimulates erythropoiesis and regulates iron metabolism. Roxadustat induces the 
expression of EPO, EPO receptors, and proteins that promote intestinal absorption of iron and recycling of iron from the macrophage iron storage system (30). A previous study showed that roxadustat treatment could correct anemia and maintain hemoglobin levels in cases where the ferritin saturation was low, the ferritin level progressively decreased, and an intermediate dose of oral iron was used (13). In this present observational research, we found no significant change in ferritin levels in any of the 21 patients after 10 weeks of roxadustat treatment. This is because we did not control iron intake during the observation study. Among the six patients with no response, two were treated with roxadustat combined with iron supplements. Among the 15 patients with an adequate response, seven were treated with iron supplements, while eight patients did not receive iron supplements. It can be seen that the therapeutic effect of roxadustat was not affected by iron status. However, due to the insufficient sample size, short follow-up time and the lack of a parallel control group or intervention, more randomized controlled trials are needed to prove the long-term safety and effectiveness of roxadustat in renal transplant anemia patients.

\section{Conclusions}

Roxadustat, as a HIF-PHI, can significantly improve hemoglobin levels and demonstrates good safety, with stable renal function and no rejection in renal transplant anemia patients. This study provides clinical evidence and experience in the treatment of renal allograft anemia.

\section{Acknowledgments}

Funding: This project was supported by the Natural Science Foundation of Zhejiang Province (No. LY19H310008) and the Hospital Pharmacy Research Project of Zhejiang Pharmaceutical Association (No. 2019ZYYYG01).

\section{Footnote}

Reporting Checklist: The authors have completed the STROBE reporting checklist. Available at https://dx.doi. org/10.21037/apm-21-2916

Data Sharing Statement: Available at https://dx.doi. org/10.21037/apm-21-2916

Conflicts of Interest: All authors have completed the ICMJE uniform disclosure form (available at https://dx.doi. org/10.21037/apm-21-2916). The authors have no conflicts of interest to declare.

Ethical Statement: The authors are accountable for all aspects of the work in ensuring that questions related to the accuracy or integrity of any part of the work are appropriately investigated and resolved. All procedures performed in this study involving human participants were in accordance with the Declaration of Helsinki (as revised in 2013). The study was approved by Clinical research ethics committee of the First Affiliated Hospital, Zhejiang University School of Medicine (No. 2020-652) and informed consent was taken from all the patients.

Open Access Statement: This is an Open Access article distributed in accordance with the Creative Commons Attribution-NonCommercial-NoDerivs 4.0 International License (CC BY-NC-ND 4.0), which permits the noncommercial replication and distribution of the article with the strict proviso that no changes or edits are made and the original work is properly cited (including links to both the formal publication through the relevant DOI and the license). See: https://creativecommons.org/licenses/by-nc-nd/4.0/.

\section{References}

1. Zhang L, Wang F, Wang L, et al. Prevalence of chronic kidney disease in China: a cross-sectional survey. Lancet 2012;379:815-22.

2. Stauffer ME, Fan T. Prevalence of anemia in chronic kidney disease in the United States. PLoS One 2014;9:e84943.

3. Drozdz M, Weigert A, Silva F, et al. Achievement of renal anemia KDIGO targets by two different clinical strategies - a European hemodialysis multicenter analysis. BMC Nephrol 2019;20:5.

4. Shi BY, Liu ZJ, Yu T. Development of the organ donation and transplantation system in China. Chin Med J (Engl) 2020;133:760-5.

5. Kim HC, Park SB, Han SY, et al. Anemia following renal transplantation. Transplant Proc 2003;35:302-3.

6. Wu Z, Guo J, Liao L, et al. Prevalence and management of post-transplant anemia in long-term follow-up of Chinese kidney transplant recipients: a single-center report. Eur J Med Res 2013;18:45.

7. Gafter-Gvili A, Ayalon-Dangur I, Cooper L, et al. Posttransplantation anemia in kidney transplant recipients: 
a retrospective cohort study. Medicine (Baltimore) 2017;96:e7735.

8. Huang Z, Song T, Fu L, et al. Post-renal transplantation anemia at 12 months: prevalence, risk factors, and impact on clinical outcomes. Int Urol Nephrol 2015;47:1577-85.

9. Becker K, Saad M. A new approach to the management of anemia in CKD patients: a review on roxadustat. Adv Ther 2017;34:848-53.

10. Lankhorst CE, Wish JB. Anemia in renal disease: diagnosis and management. Blood Rev 2010;24:39-47.

11. Besarab A, Provenzano R, Hertel J, et al. Randomized placebo-controlled dose-ranging and pharmacodynamics study of roxadustat (FG-4592) to treat anemia in nondialysis-dependent chronic kidney disease (NDDCKD) patients. Nephrol Dial Transplant 2015;30:1665-73.

12. Minutolo R, Conte G, Cianciaruso B, et al. Hyporesponsiveness to erythropoiesis-stimulating agents and renal survival in non-dialysis CKD patients. Nephrol Dial Transplant 2012;27:2880-6.

13. Chen N, Hao C, Peng X, et al. Roxadustat for anemia in patients with kidney disease not receiving dialysis. $\mathrm{N}$ Engl J Med 2019;381:1001-10.

14. Chen N, Hao C, Liu BC, et al. Roxadustat treatment for anemia in patients undergoing long-term dialysis. N Engl J Med 2019;381:1011-22.

15. Chinese Medical Association Nephrology Branch Renal Anemia Diagnosis and Treatment Consensus Expert Group. Chinese expert consensus on the diagnosis and treatment of renal anemia (revised edition, 2018). Chinese Journal of Nephrology 201;34:860-6.

16. Locatelli F, Bárány P, Covic A, et al. Kidney Disease: Improving Global Outcomes guidelines on anaemia management in chronic kidney disease: a European Renal Best Practice position statement. Nephrol Dial Transplant 2013;28:1346-59.

17. Florit EA, Hadad F, Rodriguez Cubillo B, et al. Anemia in kidney transplants without erythropoietic agents: levels of erythropoietin and iron parameters. Transplant Proc 2012;44:2590-2.

18. Malyszko J, Oberbauer R, Watschinger B. Anemia and erythrocytosis in patients after kidney transplantation. Transpl Int 2012;25:1013-23.

19. Thongprayoon C, Khoury NJ, Bathini T, et al. Epidemiology of parvovirus B19 and anemia among kidney transplant recipients: a meta-analysis. Urol Ann 2020;12:241-7.
20. Chonchol M, Lippi G, Montagnana M, et al. Association of inflammation with anaemia in patients with chronic kidney disease not requiring chronic dialysis. Nephrol Dial Transplant 2008;23:2879-83.

21. Lim JH, Jeon Y, Yook JM, et al. Medium cut-off dialyzer improves erythropoiesis stimulating agent resistance in a hepcidin-independent manner in maintenance hemodialysis patients: results from a randomized controlled trial. Sci Rep 2020;10:16062.

22. Inrig JK, Bryskin SK, Patel UD, et al. Association between high-dose erythropoiesis-stimulating agents, inflammatory biomarkers, and soluble erythropoietin receptors. BMC Nephrol 2011;12:67.

23. Provenzano R, Besarab A, Sun CH, et al. Oral hypoxiainducible factor prolyl hydroxylase inhibitor roxadustat (FG-4592) for the treatment of anemia in patients with CKD. Clin J Am Soc Nephrol 2016;11:982-91.

24. Besarab A, Chernyavskaya E, Motylev I, et al. Roxadustat (FG-4592): correction of anemia in incident dialysis patients. J Am Soc Nephrol 2016;27:1225-33.

25. Li SY, Shen B, Liu ZH, et al. Human parvovirus B 19-induced pure red cell aplasia in patients with allograft renal transplantation. Progress in Modern Biomedicine 2012;12:2698-702.

26. Yan Z, Xu G. A novel choice to correct inflammationinduced anemia in CKD: oral hypoxia-inducible factor prolyl hydroxylase inhibitor roxadustat. Front Med (Lausanne) 2020;7:393.

27. Rogo LD, Mokhtari-Azad T, Kabir MH, et al. Human parvovirus B19: a review. Acta Virol 2014;58:199-213.

28. Pan SY, Chiang WC, Chen YM. The journey from erythropoietin to 2019 Nobel Prize: Focus on hypoxiainducible factors in the kidney. J Formos Med Assoc 2021;120:60-7.

29. Griffioen AW, Bischoff J. Oxygen sensing decoded: a Nobel concept in biology. Angiogenesis 2019;22:471-2.

30. Xu MM, Wang J, Xie JX. Regulation of iron metabolism by hypoxia-inducible factors. Sheng Li Xue Bao 2017;69:598-610.

Cite this article as: Li J, Ma K, Wang L, Qi H, Lv J, Rao Y, Peng $W$. Efficacy and safety of roxadustat in the treatment of renal allograft anemia patients: a case series. Ann Palliat Med 2021;10(11):11859-11867. doi: 10.21037/apm-21-2916 